\title{
La contextualización artística del P. Pedro José Márquez en el jesuitismo expulso*
}

\author{
Antonio Astorgano Abajo ${ }^{* *}$
}

Entre los más de cinco mil jesuitas que expulsó Carlos III en 1767 de sus reinos, una docena se dedicaron a "profesiones artísticas” y escribieron sobre música, pintura y arquitectura. No se conocen estudiosos de la Escultura entre los jesuitas expulsos, como si les bastasen los estudios de Winkelman. Gozan de cierta fama musicólogos como Antonio Eximemo y Esteban de Arteaga, sin embargo dos de los que más ahínco pusieron en el estudio del arte grecolatino en pleno Neoclasicismo, el mexicano Pedro José Márquez (1741-1820) en arquitectura, y el aragonés Vicente Requeno (1743-1811) en música, pantomima, telegrafía óptica y pintura, han estado mucho más olvidados.

Curiosamente acaban de aparecer casi simultáneamente dos libros monográficos sobre cada uno de ellos, que serán referencia obligada por una larga temporada. Nos referimos a los volúmenes Vicente Requeno, jesuita y restaurador del mundo grecolatino (Prensas de la Universidad de Zaragoza, 2012) y a este Clasicismo en la época de Pedro José Márquez, coordinados respectivamente por Antonio Astorgano y Oscar Flores Flores (Figs. 1 y 2). Tomando como pretexto la lectura y comentarios sobre estas dos espléndidas monografías, redactaremos unas breves observaciones, contextualizándolas dentro de la literatura de los jesuitas expulsos y poniendo de relieve sus divergencias y paralelismos vitales y artísticos.

Puesto que estamos presentando el libro sobre El clasicismo en la época de Pedro José Márquez, nos limitaremos a hacer unas breves reflexiones sobre el contexto jesuítico novohispano que rodeó a este notable arqueólogo e historiador de la arquitectura, verdaderamente obsesionado por restaurar el mundo artístico grecolatino.

En los dos únicos lugares de Iberoamérica en los que pudo implantarse la Compañía de Jesús, inmediatamente después de su restauración universal por el papa Pío VII (agosto de 1814) fue en España y en México, lo cual no sólo se debió al número de supervivientes de la Antigua Compañía, suprimida en 1773, sino también a la buena memoria que durante casi medio siglo supieron mantener,

\footnotetext{
* Presentación del libro El clasicismo en la época de Pedro José Márquez (Coord. Oscar Flores Flores) en la Real Academia de San Fernando., el 14 de octubre de 2014.

* * Catedrático jubilado de Literatura Española. Universidad de Zaragoza. Real Sociedad Bascongada de Amigos del País.
} 
principalmente con la pluma, sus intelectuales y escritores, entre los que se encuentran dos eminentes estudiosos de las artes grecolatinas y restauradores de la Nueva Compañía de Jesús, el aragonés Vicente Requeno y el mexicano Pedro José Márquez.
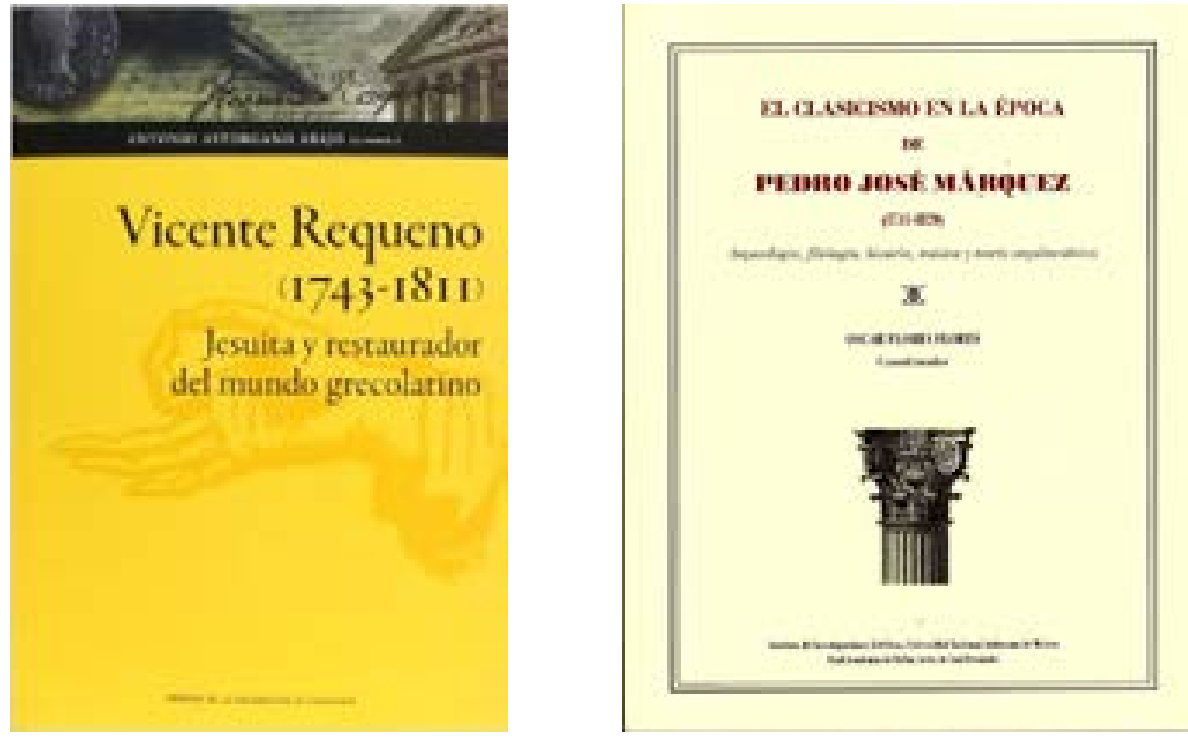

Fig. 1 y 2 Portadas de los libros sobre Vicente Requeno y Pedro José Márquez

Ambos fueron socios de mérito de la Real Academia de San Fernando y de la Real Academia de San Luis de Zaragoza, si bien no está demostrado que el P. Márquez llegase a ser admitido en la Corporación zaragozana, a pesar de su ardiente deseo, manifestado en la portada y en el prólogo de su libro, Dell' ordine dorico, ricerche dedicate alla Reale Accademia di S. Luigi di Saragoza, Roma, Salomoni, 1803.

En numerosos estudios sobre la literatura de los jesuitas expulsos, empezando por el global de la Biblioteca jesuítica Española de Lorenzo Hervás y Panduro, hemos hecho las clasificaciones cronológica y temática de la producción literaria de las diversas provincias jesuíticas desterradas.

Invariablemente hemos observado que, aproximadamente, un $10 \%$ del total de los expulsos dejó algún escrito y un 1\% redactó obras literarias de relevancia cultural en la Europa de la Ilustración. Así, de los casi 5500 jesuitas españoles expulsos, salieron unos 600 escritores, y, de estos, unos 60 con obras que todavía atraen la atención del lector del siglo XXI, entre ellos Vicente Requeno y Pedro José Márquez. Si trasladamos esta proporción a los jesuitas expulsos mexicanos, cabría esperar que de los 650 exiliados de Nueva España deberían haber salido unos setenta escritores, de los cuales una decena tendría obras relevantes.

En efecto, nos encontramos con que se cumplen bastante esas expectativas, pues, Hervás puede reseñarnos hasta 1794, 63 escritores entre los jesuitas mexicanos expulsos, de los cuales importantes son media docena, relevancia reflejada en la extensión que tienen los respectivos artículos, entre los cuales incluye a Pedro José Márquez, aunque de una manera muy extraña, es decir, no en el catálogo I (el de autores con obra publicada) sino en el catálogo II (el de autores con obra inédita), si bien pone una nota en la que dice que Pedro José Márquez debería estar entre los 
autores con obra publicada.

Nos llamó la atención el posible “despiste u olvido" de Hervás, que controlaba perfectamente lo que escribían sus compañeros, entre otras cosas porque estaba redactando su Biblioteca Jesuítica Española, cerrada en los primeros meses de 1794, aunque con pocas añadiduras hasta 1799.

Pero la extraña clasificación que Hervás dio al P. Márquez tenía una justificación irrefutable: la muy tardía aparición de la primera obra artística impresa del mexicano en 1795 (anteriormente sólo había publicado unas tablas sobre Astronomía en Roma, 1790), cuando contaba ya 55 años de edad. Surge así el primer rasgo diferencial con Requeno, quien empezó a publicar a los 41 años, con la primera edición de sus Saggi sobre el encausto (Venecia, 1784). En cuanto al final de sus carreras, pudiéramos decir, que murieron con las botas puestas, ya que Requeno (fallecido en febrero de 1811) publica sus Observaciones sobre la imprenta a mano el año anterior, mientras que Márquez trabajó hasta poco tiempo antes de su muerte en varias obras inéditas, entre las cuales las más importantes fueron los Apuntamientos por orden alfabético pertenecientes a la arquitectura donde se expone varias doctrinas de Vitruvio, y el Catálogo de los sujetos de la Compañía de Jesús que formaban la Provincia de México, formado en Roma por don Rafael Zelis, que había quedado incompleto, por su temprana muerte en Bolonia en 1798.

En 1767 la Provincia jesuita de México estaba compuesta exactamente por 678 miembros, nacidos entre 1686 y 1751, de los cuales un centenar aproximadamente dejó algún escrito literario, como hemos antes apuntado. Para hacernos una idea de los escritores surgidos en cada "generación” hemos agrupado por décadas los escritores venidos al mundo en cada una de ellas, y llegamos a la conclusión de que, en el devenir de la agitada vida de la Provincia mexicana expulsa, todas las "generaciones" o décadas dieron sus mejores frutos en el periodo 1774-1790, capitaneados por un puñado de plumas ilustres nacidas hacia 1730, que alcanza su madurez ignaciana en la profesión solemne del cuarto voto del 15 de agosto de 1763 y del 2 de febrero de 1765, cuando profesaron, entre otros, Diego José Abad, Agustín Pablo de Castro, Manuel Mariano de Iturriaga, Francisco Javier Alegre, Francisco Javier Clavijero y Rafael Landívar, donde encontramos los nombres más significativos de la literatura mexicana exiliada.

Es la generación que llega a Italia con plenitud vital (entre 35 y 40 años), que habían recibido una sólida formación humanística de maestros que no sobrepasaban los 45 (el más significativo es Rafael Campoy, nacido en 1723). Es la que hemos llamado "Generación Dorada” de la literatura expulsa mexicana, la de la década de los nacidos entre 1731 y 1740, de la que llegaron al exilio 197 jesuitas, de la que surgieron, al menos, 28 escritores.

Pedro José Márquez está incluido en la generación más joven, la de los nacidos entre 1741 y 1751, de la que llegaron 115 al exilio y de la que surgieron diez escritores, de bastante relevancia, entre los que debemos recordar al filósofo moderno y ecléctico Andrés de Guevara y Basoazábal, cuyo Prodromus ad Institutiones philosophiae y sus adaptaciones para uso de estudiantes fue libro de texto bastante utilizado durante el primer tercio del siglo XIX. 
Un rasgo común de los jesuitas jóvenes novohispanos de la generación de Pedro José Márquez fue su mexicanidad y deseo de retornar a su tierra, con diversa suerte. Por eso, entre 1798 y 1801, varios ignacianos mexicanos se asentaron en puertos españoles a la espera de su embarque para Nueva España. Nos consta que el P. Márquez, el 5 de septiembre de 1798 estaba en Florencia, rumbo a España, según carta a Azara, contándole que un corsario le había robado la librería (biblioteca). El 9 de enero de 1800 estaba en Valencia, según carta a Azara, en la que le comunica que ya había acabado el libro Dell' ordine dorico, que publicará en Roma en 1803, dedicado a la Real Academia de San Luis, de la que Azara era protector. No cabe duda que el embajador Azara, que había sido el padrino para ingresar en la Academia de San Fernando, también lo era para su posible ingreso en la Academia de San Luis, y al fallecer éste en París el 24 de enero de 1804, el proyecto se vino abajo. Sin duda, Valencia fue el punto más cercano a las Academias de San Fernando y de San Luis de Zaragoza, en cuya Academia deseaba ingresar. En el 1801 fue obligado a retornar a Italia (Fig. 3).

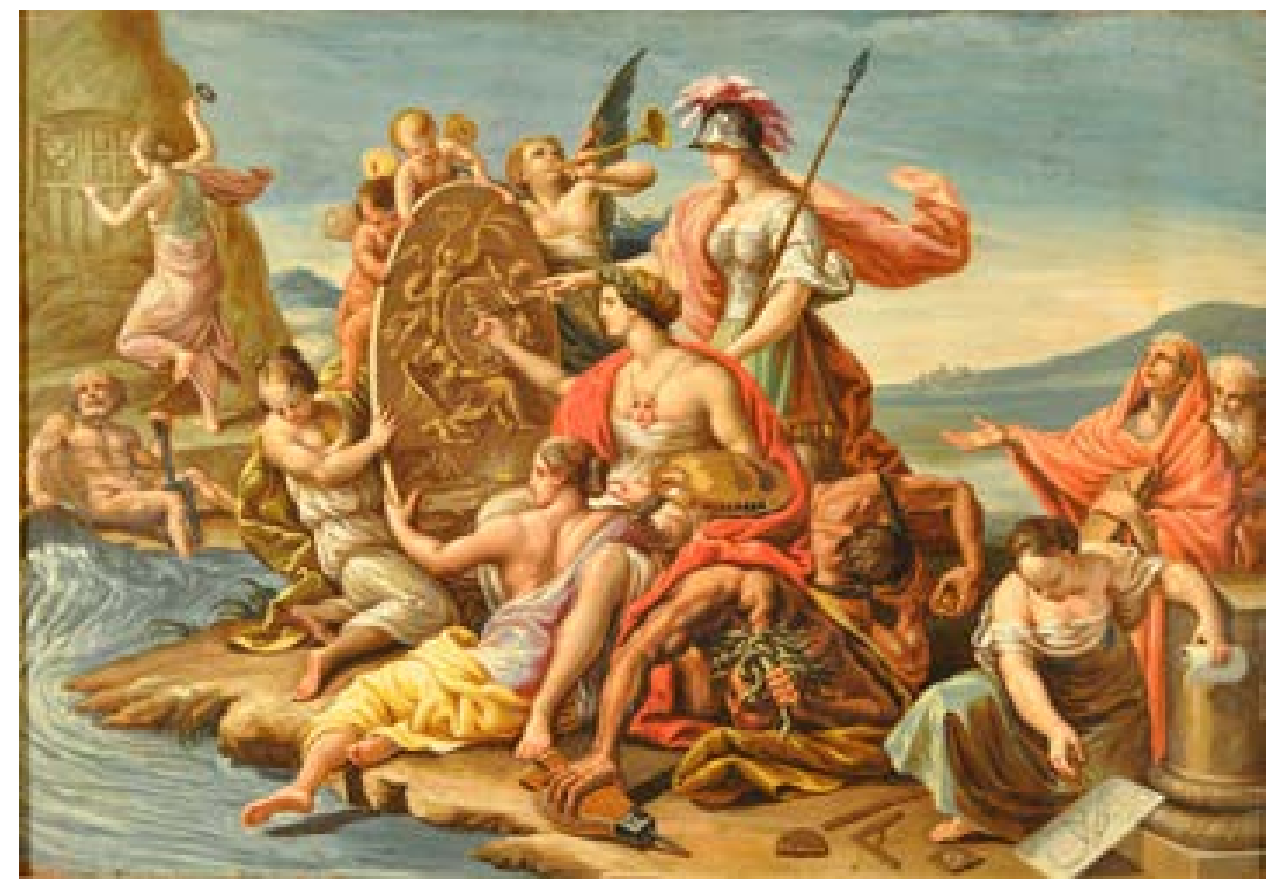

Fig. 3 Pedro José Márquez no consiguió ser admitido en la Real Academia de Nobles y Bellas Artes de San Luis de Zaragoza. Por el contrario, Requeno ingresó en dicha Real Academia, en diciembre de 1799, regalándole, como obra de entrada, La Alegoría de las tres Bellas Artes, encausto sobre tabla de fray Manuel Bayeu (59x83,50 cm. Colección de la Real Sociedad Económica Aragonesa de Amigos del País), realizado bajo "la instrucción y método” de Vicente Requeno, restaurador de este método pictórico de los antiguos griegos y romanos.

Más suerte tuvieron los novohispanos Juan Luis Maneiro, conocido biógrafo y latinista, y los provinciales, José María Castañiza y Pedro Cantón, que lograrán llegar a México en 1809.

Ese mexicanismo llevará a los expulsos novohispanos a escribir sobre temas de su tierra. Maneiro es fuente fundamental para conocer la literatura de la provincia jesuítica mexicana, pues retrató con detalle 35 jesuitas expulsos mexicanos incluidos 
en De vitis aliquot mexicanorum (Vidas de algunos mexicanos ilustres), cuyos tres tomos fueron reseñados por el P. Luengo, con cierto disgusto por haber Maneiro ocultado en el título la procedencia jesuítica de los biografiados, aludiendo exclusivamente a su nacionalidad mexicana.

Son, sin duda, estos aspectos biográficos de los expulsos, junto con su defensa del hombre americano, que aparecen en escritos de tipo etnográfico e histórico con la finalidad de establecer el grado de civilización alcanzado por los indígenas, y demostrar la existencia de una historia anterior a la conquista, tan digna y heroica como la de Hernán Cortés, los que más continúan atrayendo el interés del lector del siglo XXI. Aquí se encuadran obras de análisis artístico como la Interpretazione del codice borgiano de José Lino Fábrega, o la descripción en italiano de la pirámide del Tajín y las ruinas precolombinas de Xochicalco de Pedro José Márquez.

Será el historiador del arte hondureño, pero adscrito a la Provincia de México, Lino Josef Fábrega Bustamante (fallecido en 1797), el ex jesuita con el que más paralelismo vital presenta Pedro José Márquez, pues ambos, tras la extinción de la Compañía se retiraron a Roma, donde contaron con la protección de importantes personajes contrarios a la Compañía de Jesús, el P. Márquez con el conocido mecenazgo del embajador José Nicolás de Azara, y Lino José Fábrega, con el del cardenal Stefano Borgia, prefecto de la Propaganda Fidei, gracias a lo cual pudieron dedicarse al estudio de las antigüedades grecorromanas y mexicanas, en el caso de Márquez, y mexicanas exclusivamente en el del P. Fábregas, quien nos dejó una importante y relevante obra para el estudio de la cultura náhuatl, la citada Esposizione del codice borgiano.

Para conseguir sus objetivos literarios no dudaron en colaborar y adular a personajes e instituciones pro gubernamentales, y por lo tanto marcadas por el anti jesuitismo estimulado por los regalistas gobernantes madrileños. Así como Márquez estuvo al servicio de Azara hasta que éste fue nombrado embajador en París, Fábrega continuaba, poco antes de fallecer, al servicio del cardenal Borgia, "famoso por su aversión para con los jesuitas, y por sus muchas y grandes hazañas contra ellos”, en palabras del P. Luengo.

Si nos fijamos en Requeno, éste coqueteó con el embajador José Nicolás de Azara entre 1784 y 1788, consiguiendo que el prestigioso impresor Bodoni le editase la segunda edición de los Saggi sobre la encáustica, y que se le concediese la pensión doble a Requeno en junio de 1785, quitándosela al padre Bartolomé Pou, también residente en Bolonia, que era quien la había solicitado directamente a Floridablanca.

Requeno sólo interrumpió su amistad con el embajador en 1788 cuando su íntimo amigo, san José Pignatelli, lo previno contra las intrigas anti jesuíticas de Azara, quien andaba indagando el autor del libro anónimo, la Segunda Memoria Católica, donde se sostenía que la supresión de la Compañía de Jesús había sido contraria a todo derecho. El padre Luengo comenta que un agente de Azara le pidió información a Requeno sobre dicha Segunda Memoria Cathólica, "pero antes de empeñarse en la cosa, se lo contó a don Joseph Piñateli, quien le aconsejó que se guardase bien de dar noticias algunas sobre aquella obra, y que procurase descargarse de aquella odiosa y peligrosa comisión”. 
No tenemos tiempo de extendernos ahora en el aragonesismo de Requeno y en sus cordiales relaciones con la Real Sociedad Económica Aragonesa y con la Real Academia de San Luis entre 1785 y 1801. Ahora solamente apuntaremos que Requeno y Márquez se respetaron escrupulosamente los respectivos campos de investigación, pues el mexicano, en las pocas veces que alude a la pintura grecolatina, se limita a decir que no va a tratar el tema, porque ya ha sido suficientemente estudiado por Vicente Requeno. Por su parte, el aragonés no trató asuntos arquitectónicos, ni hemos encontrado una referencia literaria expresa a los mismos.

Si nos fijamos en el cuadro Alegoría de las tres Bellas Artes exaltando a la Real Academia de San Luis, obra de fray Manuel Bayeu, pero en realidad atribuible en gran parte a Requeno, porque, concebida como obra de ingreso en la Real Academia de San Luis, el jesuita le dio el tema, la estructura, el ambiente y los personales al caduco fraile cartujo Bayeu, vemos que el ex jesuita aragonés da relieve a la Pìntura (escena central amplia), después a la arquitectura (escena en la parte derecha del espectador) y en último término y alejada aparece la escultura con su maza.

Dentro del cuadro ya se indica la intervención del abate de Calatorao, en la inscripción en el borde frontal de un libro que sostiene la Aritmética con la palabras: "SET / 1799", mes en el que es electo académico de honor de la de San Luis y de la de San Fernando. En el otoño de 1799, Requeno llevaba un año justo residiendo en Zaragoza ya había atraído hacia la técnica encáustica a fray Manuel Bayeu, pues la Alegoría de las Bellas Artes era el "segundo ensayo" que hacía según "la instrucción y método” del jesuita.

Respecto al simbolismo de las figuras, el abate aragonés no alude al Río Ebro en su carta de presentación a la Academia, si bien es aceptable, dado el contexto aragonesita que rodea el cuadro y las intenciones de los autores (impulsar las artes en la juventud aragonesa). En la carta de presentación se nos desvela la identidad de los personajes menores que rodean a la Arquitectura: la Aritmética (no la Ciencia o la Matemática, como han escrito la totalidad de los críticos) y el Consejo (no el Tiempo). Respecto a las otras figuras (Discordia, rey Midas, Ignorancia, etc.) nada dice el coautor Requeno, por lo que puede correr libre la imaginación de los estudiosos.

La composición del cuadro es clara pues organiza los elementos gráficos y plásticos horizontalmente en el espacio, de forma que la expresión visual tiene una coherencia formal y una lectura comprensible, transmitiéndonos sensaciones de estabilidad, afirmación y continuidad. Esta línea visual horizontal les permite a Requeno-Bayeu disponer las formas en tres escenas totalmente delimitadas y una marcadísima perspectiva, a pesar de poner en el centro del cuadro a su protagonista principal, la alegoría de la pintura.

Las formas son figurativas y cerradas, pues están definidas por líneas visibles que delimitan los contornos de los diferentes elementos, aislando unos de otros y permitiendo ver tres escenas perfectamente estructuras por separado.

Presenta una cuidada composición sobre un fondo marino, en el que aparecen diversas alegorías de las Bellas Artes, la Escultura, Pintura, Arquitectura, Aritmética 
(Filosofía), junto a la Fama, el Rio Ebro (Neptuno), Midas (Mercurio), Minerva y los Vicios a sus pies.

En esta obra, Requeno y Fray Manuel Bayeu demuestran no solo el dominio técnico alcanzado en el procedimiento pictórico del encausto sino también su habilidad para componer escenas con un elevado número de personajes. En efecto, consiguen ocupar toda la superficie del cuadro disponiendo las figuras en tres grupos humanos bien diferenciados, haciendo incidir la luz sobre las tres Bellas Artes y sobre los personajes que simbolizan las virtudes que la acompañan, poniendo, por el contrario, en oscuridad, los vicios que deberían corregirse. Es por tanto una obra que tiene un mensaje pedagógico y por tanto proyecta valores positivos, que dejan en el espectador sensaciones alegres por encima del misterio del simbolismo, que no es nada complicado.

En el centro, una alegoría de la Pintura está dando las últimas pinceladas a una medalla ovalada en cuyo interior se ha representado el escudo de la R.S. Económica Aragonesa o de la R. Academia de San Luis, que son muy parecidos; siendo guiada en esta delicada tarea por Minerva, diosa de las Artes. Completan este grupo central un personaje con orejas de burro, que, según unos es el dios del Comercio, Mercurio, y según otros el rey Midas, que suele representarse con orejas de burro y con la manzana de oro en la mano. Otra monstruosa figura de un Vicio, una alegoría de la Fama, y en último lugar, dos querubines y unas jóvenes que están sosteniendo la medalla a la Pintura.

En el lateral izquierdo, una representación femenina de la Escultura talla el Escudo de Aragón en un peñasco de mármol en la ladera de una montaña, mientras que una alegoría del Ebro deja fluir su abundante caudal que emana de una vasija en esa misma montaña.

En el lateral derecho, figura una personificación de la Arquitectura y un personaje femenino que personifica a la Aritmética y otro masculino que simboliza el Consejo, según identificación del mismo Requeno, pero que la crítica hasta ahora se ha limitado a definirlos como la Sabiduría.

Resulta evidente, por tanto, que Requeno, restaurador de la técnica pictórica grecolatina del encausto y pintor aficionado, y fray Manuel Bayeu, pintor profesional, han querido subrayar la preeminencia de la Pintura sobre las demás Artes. La manera que tuvieron de sugerir la superioridad de esta disciplina artística fue presentarnos a la Pintura sentada. En el caso del cuadro que nos ocupa, consideramos que además de proclamar la supremacía de la Pintura, Requeno y fray Manuel Bayeu exponen su parecer en relación a la jerarquía de las Artes, en el que la Pintura ocuparía el primer puesto y la Escultura, el último. Lógicamente el jesuita mexicano Márquez había pintado un cuadro con la Arquitectura en el lugar que Requeno le otorga a la Pintura.

Sin duda ambos jesuitas nos ofrecerían un abigarrado conjunto de figuras alegóricas, con personajes principales y menores, puesto que dominaban la compleja iconografía grecolatina, después de treinta años de lectura continua de autores clásicos. Si se analizan de forma individual las figuras y los detalles de la Alegoría de las tres Bellas Artes, se advierte rápidamente que Vicente Requeno y fray Manuel Bayeu quisieron dotar de un profundo sentido simbólico al cuadro, haciéndose visible su hondo conocimiento de la Iconología del académico Cesare Ripa Perugino 
(c. 1555-Roma, 22-enero de 1622) ${ }^{1}$. Requeno-Bayeu se preocuparon de representar los personajes, sobre todo las figuras de los dioses, siguiendo su iconografía más conocida y extendida con el fin de favorecer su inmediata identificación. Es el caso inconfundible de la diosa Minerva y del rey Midas. Nos parece interesante destacar que tanto Minerva como Midas protagonizan dos mitos que fueron utilizados como argumento en la defensa de la superioridad del arte sobre la artesanía y de la condición divina de las bellas artes.

\section{Escena central de la Pintura}

No vamos a detenernos en la descripción de las tres escenas (Pintura, Arquitectura y Escultura). Aparentemente la escena de la pintura (Fig. 4) desequilibra la composición del cuadro en el campo visual, que adquiere un peso decisivo en la distribución de los distintos pesos. Por su posición ocupa la centralidad. Por su tamaño abarca más de la mitad del espacio y por sus colores contiene los más cálidos de toda la obra, destacando el rojo vivo del manto que cubre al personaje de la Pintura. Por ello, el mayor peso visual de esta escena es evidente. Sin embargo encontramos cierta simetría axial en torno a un eje imaginario constituido por las tres figuras femeninas escalonadas que rodean la figura de la pintura, lo que da la sensación de estar el cuadro dividido en dos partes simétricas.

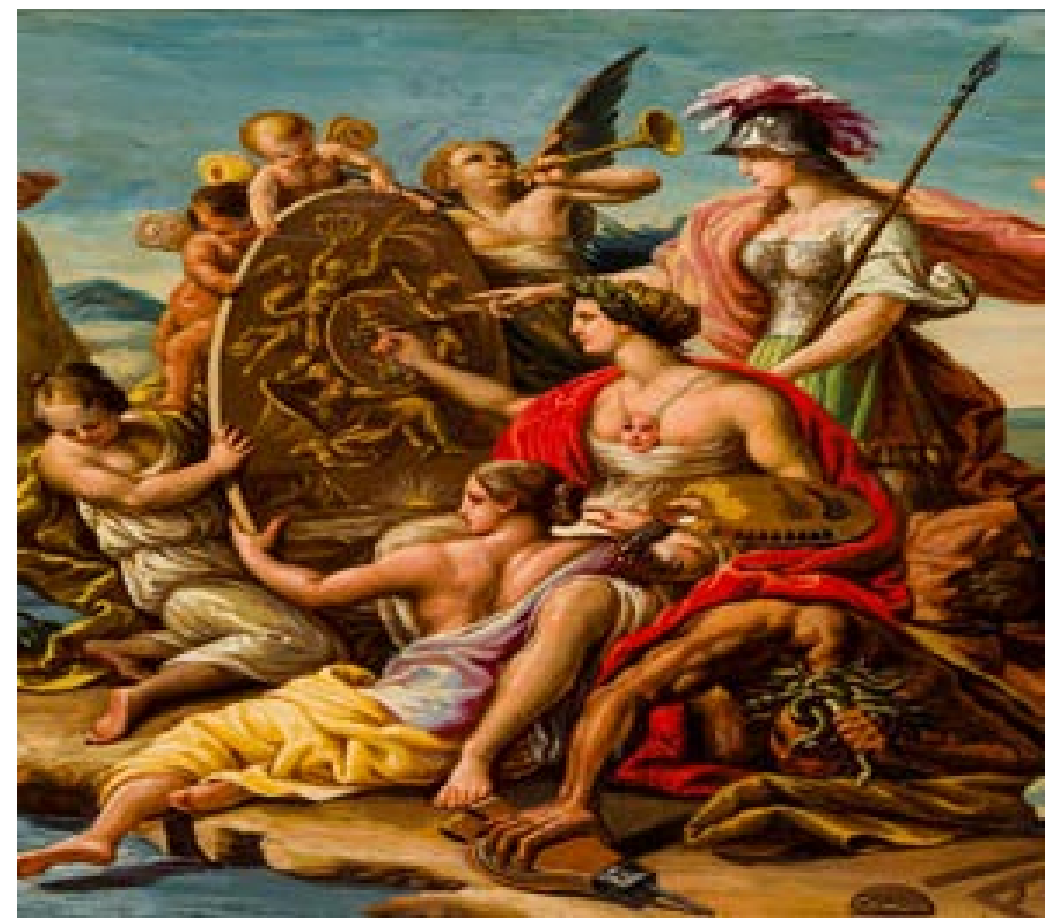

Fig. 4 Escena central de la Pintura

\footnotetext{
${ }^{1}$ Cesare RIPA PERUGINO, Iconología, Madrid, Akal, 1987, 2 vols. Edición y estudio introductorio por Adita Allo Manero. La utilización de la obra de Ripa por parte de Fray Manuel Bayeu ha quedado sobradamente demostrado en los siguientes trabajos: CALVO RUATA, J. I., "La alegoría como tema pictórico en la Cartuja de Nuestra Señora de las Fuentes (Huesca)”, AnalectaCartusiana, n 3, 1990, pp. 79-97, y ESTEBAN LORENTE, J. F., “Goya. De la alegoría tradicional a la personal”, Artigrama, $\mathrm{n}^{\circ}$ 25, 2010, 103-121, espec. pp. 104-106.
} 
El equilibrio de la estructura compositiva se consigue en torno a la figura de la Pintura, que por ser la más importante ocupa el centro jerárquico y visual, consiguiendo ser el principal foco de atención de la obra. A ambos lados de ella se disponen dentro de la misma escena arriba y abajo, adelante y detrás, otras figuras de carácter secundario y semejante peso visual, que hacen de contrapunto a la figura principal. Asimismo las escenas de la Arquitectura y la de la Escultura equilibran, en cierta media, el dominio de la escena de la Pintura que de otra manera nos parecería apabullante, por lo que podemos afirmar que los pesos visuales de todos los elementos y formas o manchas, de la obra son semejantes y equilibrados. La composición resulta coherente y lo que domina es una composición armónica en formas, texturas y color. Fray Manuel Bayeu y Requeno han querido realzar la importancia de esta figura situándola en el lugar más importante, ciñéndole en la cabeza una corona de laurel, cubriéndola con un majestuoso manto rojo y representándola en actitud sedente. En ningún caso, Ripa recomienda representar a la Pintura con la corona de laurel, distintivo que, sin embargo, Requeno-Bayeu le conceden para aludir a la fama y a la gloria que alcanzan los practicantes de este noble arte. Este detalle de la corona de laurel creemos que debió ser una sugerencia de Vicente Requeno, ya que su simbología se encuentra a cada paso en los autores clásicos. Por su parte, el manto rojo que cubre a la mujer, al ser un atributo reservado para la monarquía, es símbolo de la soberanía y una señal de dignidad superior. Incide en esta misma idea de superioridad y de nobleza el hecho de que la Pintura se muestre sentada.

\section{Escena de la Arquitectura}

Requeno sabía muchísimo menos de Arquitectura que Márquez, pero el abate aragonés había estudiado en profundidad a Vitrubio (autor primordial para Márquez), una de las escasísimas fuentes conservadas sobre la técnica pictórica del encausto. Requeno le concede el segundo lugar en la jerarquía de las Bellas Artes. A la derecha del cuadro, agachada en primerísimo plano, está la alegoría de la Arquitectura, de nuevo una mujer, trazando líneas con un compás sobre un papel en el que hay varias figuras geométricas. Apoya el otro brazo sobre una columna, elemento constructivo esencial. A sus pies hay otros instrumentos propios de la Arquitectura, que son un graduador, una escuadra o cartabón, una regla y una brújula (Fig. 5). 


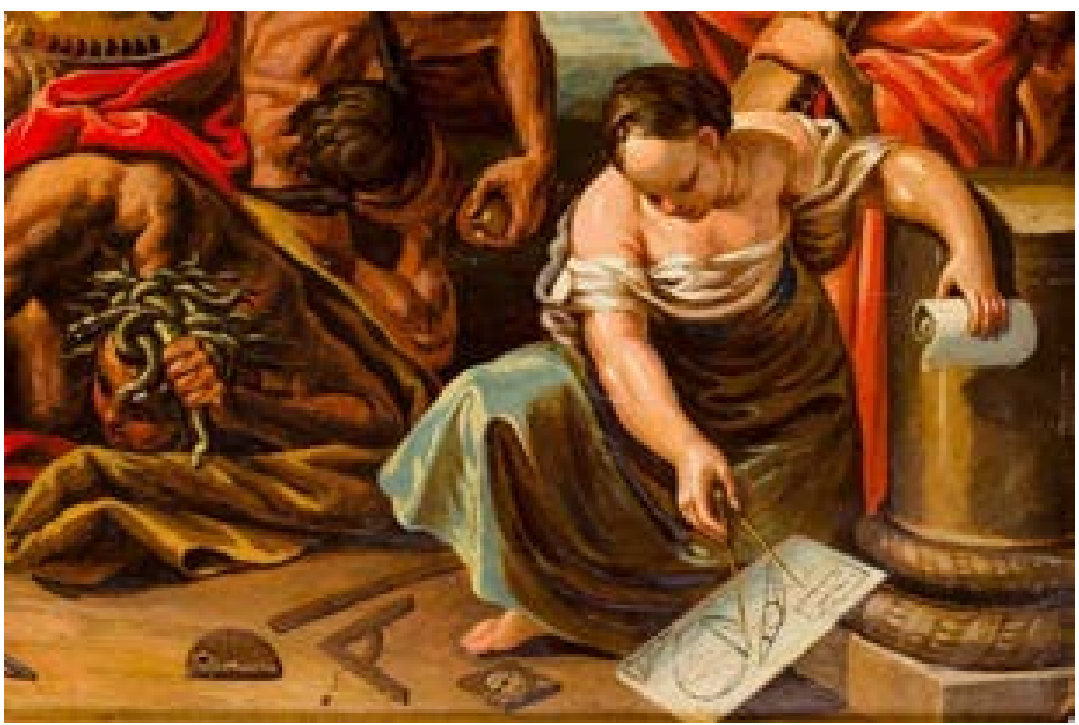

Fig. 5 Escena de la Arquitectura

Ripa, siguiendo a autores clásicos como Vitrubio y Platón, sostiene que la Arquitectura es una ciencia y un arte muy superior al resto por participar de la Aritmética y Geometría. Por esta razón, recomienda que junto a la alegoría de la Arquitectura aparezcan una escuadra y un compás por ser los instrumentos propios de la Geometría, y que en la planta arquitectónica que está dibujando figuren unos números en alusión a la Aritmética, indicación esta última que en la obra de Requeno-Bayeu no se cumple en el pergamino, pero sí en la tarjeta de la cabeza.

En esta escena aparecen otros dos personajes o figuras alegóricas, identificadas por Requeno en la carta de presentación del cuadro: la Aritmética y el Consejo (Fig. 6); la primera de ellas, una mujer cubierta con una toca o manto, que muestra sobre su cabeza una tarjeta con guarismos o signos ininteligibles, representa la Aritmética, como indica el mismo Requeno en la carta de presentación. No sabemos por qué Jonathan Brown y José Luis Morales y Marín la identifican con una visualización de la Filosofía, puesto que la Aritmética es más útil que la Filosofía para el arquitecto, máxime si ya cuenta con el "El Buen Consejo" que es la figura que la asesora. En la mano izquierda sostiene un libro, en cuyo borde central lleva la leyenda "SEP. 1799", el mes en que Requeno fue nombrado académico de honor de la Academia de San Luis, como hemos dicho, y sin duda el mes en que se comenzó la ejecución del cuadro. 


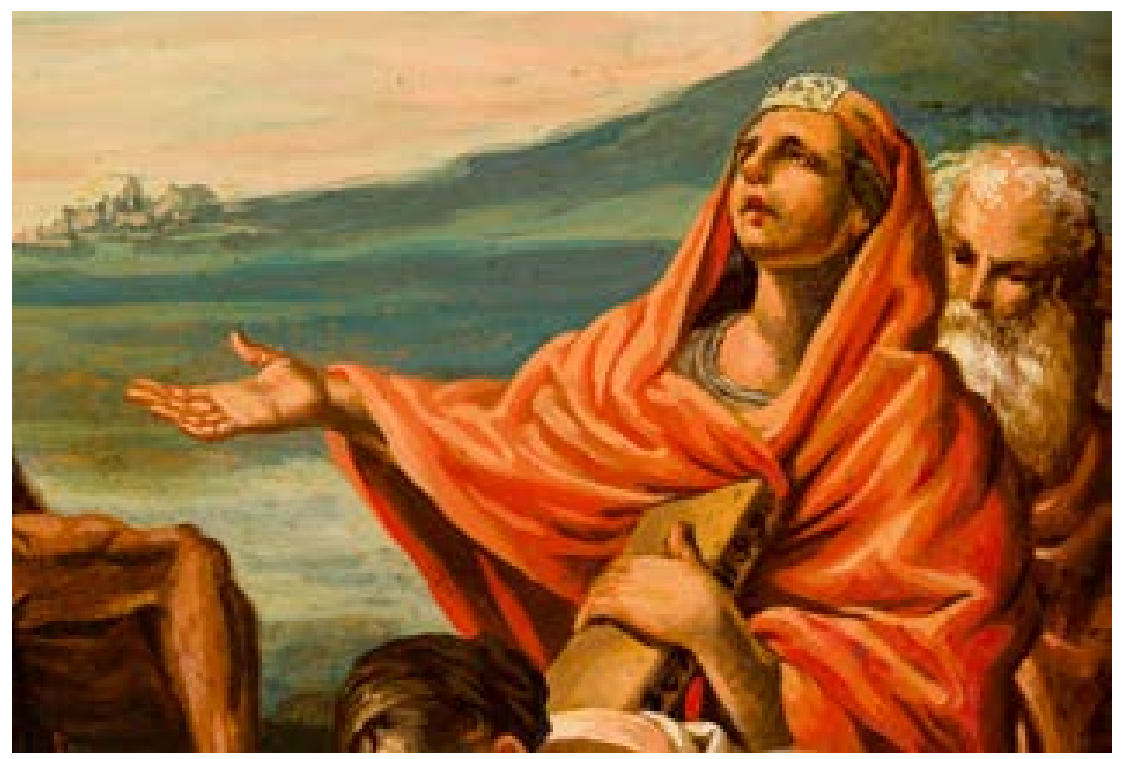

Fig. 6 Detalle de las figuras de la Aritmética y el Consejo

El desconcierto de la crítica respecto a la otra figura, la cabeza de un anciano, ha sido total, llegando algunos, como Ricardo Centellas, a identificarla con el Tiempo. Sin embargo Requeno nos dice que simboliza el buen Consejo, sin duda virtud muy necesaria a un buen arquitecto para evitar proyectos descerebrados, como ha puesto de manifiesto la última burbuja inmobiliaria en España. Cesare Ripa y los clásicos representaban la virtud de la sensatez en el arquitecto mediante un péndulo o plomada para señalar que el buen arquitecto debe estar siempre atento a la consideración de su centro. Requeno y Bayeu deciden cambiar el péndulo por todo un personaje que simbolizase la sensatez que debe tener un buen arquitecto, y lo llama "El Consejo", en forma de viejo venerable, que nos recuerda al "admonitor" o asesor que San Ignacio de Loyola colocó a lado de todo superior para su mejor gobierno.

\section{Escena de la Escultura}

En el extremo izquierdo de cuadro, una mujer que simboliza la Escultura (Fig. 7) está tallando con martillo y cincel el escudo del Reino de Aragón en una gran roca. En comparación con la Arquitectura y la Pintura, la alegoría de la Escultura resulta de una sencillez inusitada. Vuelta de espaldas, ocupa el lugar más alejado respecto al punto de vista del espectador, portando como únicos atributos un cincel y un mazo que la identifican como la personificación de este Arte. En esta escena hay un personaje secundario, el Río Ebro, debajo de la Escultura. Casi toda la crítica coincide en que la figura de un anciano desnudo vertiendo abundante agua con un cuerno personifica al río Ebro. 


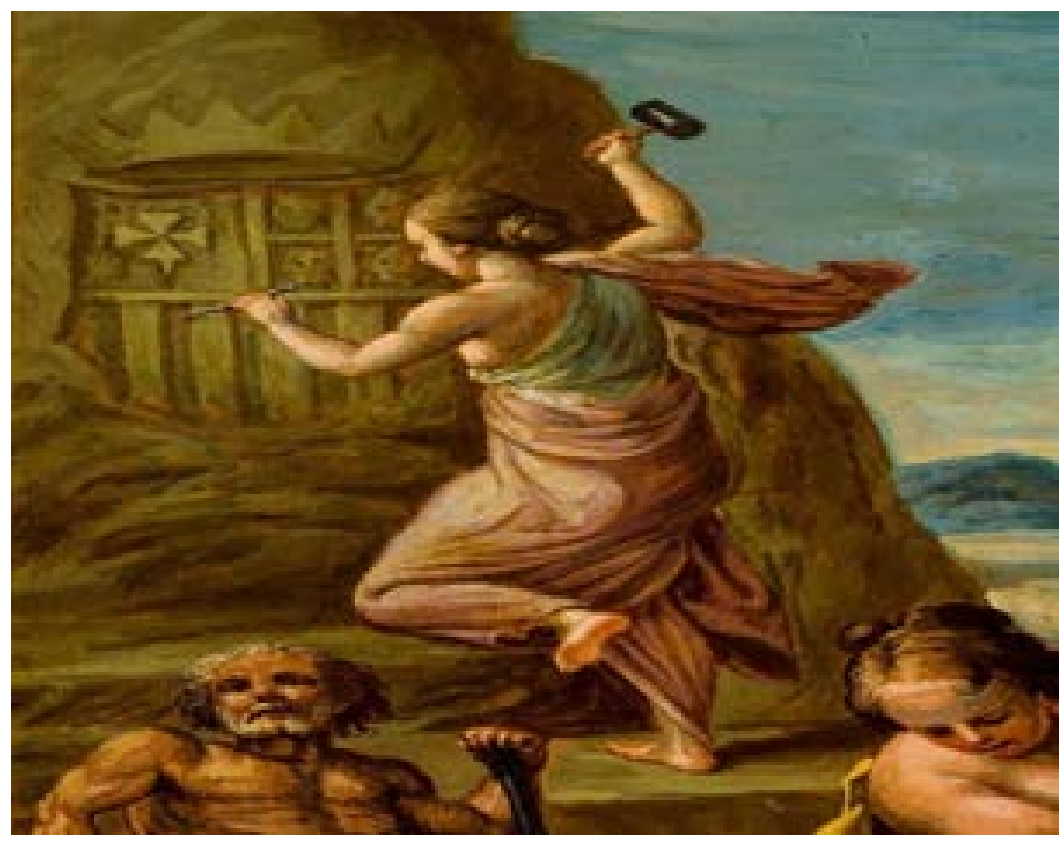

Fig. 7 Escena de la Escultura

En síntesis, el cuadro representa alegóricamente a las tres artes plásticas mayores impartidas en la Academia de San Luis de Zaragoza, al amparo de su diosa protectora, que con su gesto exalta el emblema de esa Real Academia, implantada en Zaragoza en 1792 para mayor florecimiento del pueblo aragonés y mejor superación de lacras humanas, como son la Discordia y la Ignorancia. La presencia del Ebro y del escudo de Aragón refuerza el particular sentido geográfico del conjunto alegórico.

La Alegoría de las tres Bellas Artes es todo un testimonio de adhesión al más puro idealismo clasicista, tanto por la técnica como por la iconografía y el estilo, que atribuimos exclusivamente a Vicente Requeno, un auténtico obseso por restaurar las artes grecorromanas.

En suma, el cuadro está impregnado de espíritu ilustrado, del que siempre careció nuestro fraile. Tanto por la carta con que Requeno presenta el cuadro a la Academia de San Luis como por sus constantes investigaciones para restablecer las artes grecolatinas, esta obra es un homenaje a la Real Sociedad Económica Aragonesa, y más en concreto a los socios que estaban al frente de la Academia de San Luis, y un elogio de las tres Bellas Artes, en especial de la Pintura, a la que fray Manuel Bayeu y Vicente Requeno consideraban como la disciplina artística superior, y a la que entregaron apasionadamente sus vidas.

Esta contextualización artística del único cuadro del que nos consta la coautoría de Requeno, nos lleva a ver notables coincidencias con su correligionario mexicano. Pedro José Márquez tuvo relación con la Económica Aragonesa y con la Real Academia de San Luis, aunque no aparece mencionado ni una sola vez en las actas correspondientes. Nos basamos en el hecho de que en los fondos de la Biblioteca de la Económica Aragonesa encontramos los siguientes títulos: Delle case di cità degli antichi Romani, 1795, dedicado a la Real Academia de San Fernando, encuadernado en pergamino; Delle ville di Plinio, 1796, dedicado a José Nicolás de Azara. 
El más serio intento de acercarse a la Academia de San Luis lo realizó el P. Márquez en 1803, dedicándole a dicha Academia Del Ordine dorico, cuya licencia de impresión está fechada en Roma, el 21 de junio de 1803. Al efecto envió a la Academia cuatro ejemplares magníficamente encuadernados, que se conservan en la Biblioteca de la Real Sociedad Económica Aragonesa, que era donde se depositaban los enseres de la Academia de San Luis, dado que los directivos de ambas instituciones eran los mismos. Por ejemplo, Juan Martín de Goicoechea, el íntimo amigo de Goya, era vice director de la Económica Aragonesa y segundo consiliario de la Academia de San Luis.

Lo sorprendente es que Pedro José Márquez, que tan celosamente perseguía los honores académicos, en las Investigaciones sobre el orden dórico, simplemente se autodefine como "mexicano", sin aludir a su pertenencia como socio de honor a la Real Academia San Fernando (Fig. 8).

A pesar del empeño que Márquez puso en agradar a la Academia de San Luis y a la Sociedad Económica Aragonesa, que en esta época mantenían una estrecha relación paterno-filial, no consiguió que se le tomase en serio en Zaragoza, pues la primera no lo hizo académico y no aparece mencionado ni una sola vez en los Libros de Resoluciones o actas de la Económica Aragonesa, como hemos dicho.

Intentando buscar alguna explicación a este desencuentro, la hemos hallado en que tanto la Academia de San Luis como la Económica Aragonesa, hacia 1803 tenían una visión de la arquitectura mucho más práctica y utilitaria que las propuestas arqueológicas grecolatinas del P. Márquez.

El arquitecto de referencia de ambas instituciones era el director de Arquitectura de la Academia, Francisco Rocha Cabrera (Monzón, 1766-Zaragoza, 1809), quien implantó en Zaragoza un neoclasicismo racional y funcionalista realizando proyectos, visuras y peritaciones, un tanto alejadas de las exquisiteces grecolatinas.

Ciertamente eran muchas y acuciantes las ocupaciones que agobiaban a los dos profesores arquitectos de la Academia de San Luis (Inchauste y Rocha), requeridos para planificar y supervisar los más diversos proyectos de obra civil, encargados por todo tipo de autoridades. En consecuencia, los libros y aspiraciones de Pedro José Márquez pasaron a un segundo plano. Por ejemplo, en la Junta de la Económica Aragonesa del 29 de abril de 1803, el secretario de Estado, Pedro Ceballos, le encarga a Manuel Inchauste y a Francisco Rocha que "vayan a nivelar el terreno y levantar los planos del canal de Tamarite de Litera”. 
No es de extrañar que el último libro de Márquez que encontramos en la biblioteca de la Económica Aragonesa, Due antichi monumenti di Archittetura Mesicana di Pietro José Márquez, donde se autocalifica de socio de la de las academias de bellas artes de Madrid, de Florencia y de Bolonia, dedicados a la muy noble, ilustre e imperial ciudad de México, publicado en Roma en 1804, por el habitual editor Salomoni, sea un ejemplar sin encuadernar, que aún hoy permanece sin desvirgar, indicio suficiente para comprender el poco interés que el tema de la arquitectura mexicana ha suscitado entre los miembros de la Económica Aragonesa y de la Academia de San Luis durante los dos últimos siglos.

Este libro fue enviado a la Económica Aragonesa incluyendo siete ejemplares de una hoja de propaganda titulada Nota de las obras dibujadas y grabadas en Roma por Juan Bautista Cipriani hasta diciembre de 1803. Para esta fecha ya estaban a la venta las Investigaciones sobre el orden dórico de Pedro José Márquez, pues se dice que se vendía al precio de 70 bayocos.

Resumiendo, una ojeada superficial a la cronología de la literatura de los jesuitas mexicanos expulsos, nos muestra que la primera y la última década presentan menos escritores, la primera (nacidos entre 1686 y 1700) por razones de edad, y la última (nacidos entre 1741 y 1751) por las persecuciones y deserciones fomentadas por las autoridades borbónicas (recuérdese el caso de los novicios, que fueron enviados a sus casas).

Sin embargo se diferencian en la calidad de los más jóvenes (Pedro José Márquez, Guevara Bazoazábal, Lino José Fábrega, Juan Luis Maneiro...), que contrasta con los viejos teologones de principios del siglo XVIII.

Las dos décadas o generaciones centrales (nacidos entre 1720 y 1740) presentan el mismo número de escritores, con una calidad cada vez más elevada en su obra, estimulada por la nostalgia y defensa del hombre y del paisaje americanos, que culmina con los escritos de tipo poético, etnográfico e histórico de los grandes autores nacidos en la década de 1731-1740 (Clavigero, Landívar, Andrés Cavo...), que captó la cultura ilustrada, sin buscar ciegamente la inmersión en la misma, sino más bien la contradijo críticamente en su visión de la historia y antropología americanas (recuérdense las polémicas con William Robertson, Guillaume Thomas Raynal y Cornelius de Pauw).

Por su parte, los jesuitas aragoneses se distinguieron por su oposición y denuncia de una serie de prejuicios, que la Europa ilustrada tenía sobre el mundo hispánico, y que culminarían en el famoso artículo denigrativo e injusto sobre España que Masson de Morvilliers publicó en 1783 en la Encyclopédie méthodique, recuérdense el Saggio storico-apologetico della Letteratura spagnuola de Francisco Javier Llampillas, traducido por Josefa Amat y Borbón, y la Historia crítica de España y de la cultura española de Juan Francisco Masdeu, quien no podía tolerar que el español Requeno viese usurpada la paternidad de sus hallazgos artísticos (encausto, telegrafía óptica, pantomima clásica, etc.) por los extranjeros, razón por la que dijo en 1804 y publicó en 1806, su patriótico discurso, Requeno, il vero inventore delle più utili scoperte della nostra età (Fig. 9). 
La generación más joven, la de Pedro José Márquez y la de Vicente Requeno, fue la que más profundamente se sumergió en la cultura ilustrada de la Italia del momento, no dudando en buscar el mecenazgo de personajes radicalizados de la Ilustración, como el embajador José Nicolás de Azara y el cardenal Stefano Borgia. Pero nunca rompieron la vinculación con sus raíces jesuíticas, lo que le da un aire de contradicción a muchas de las biografías y de la literatura de estos jesuitas.

Es difícil de creer que un Pedro José Márquez, que estuvo bajo la protección de las Academias, eruditos y artistas ilustrados y filo revolucionarios para poder realizar su trabajo con tranquilidad, poco más tarde fuese un pilar de la restauración de la Compañía de Jesús en México. Estuvo al servicio del embajador José Nicolás de Azara durante unos quince años y no tuvo inconveniente en integrarse en la instituciones culturales bajo el dominio de los invasores revolucionarios franceses, como en la Academia Romana de Arqueología, o en aceptar en agosto de 1811 la dirección de la Biblioteca

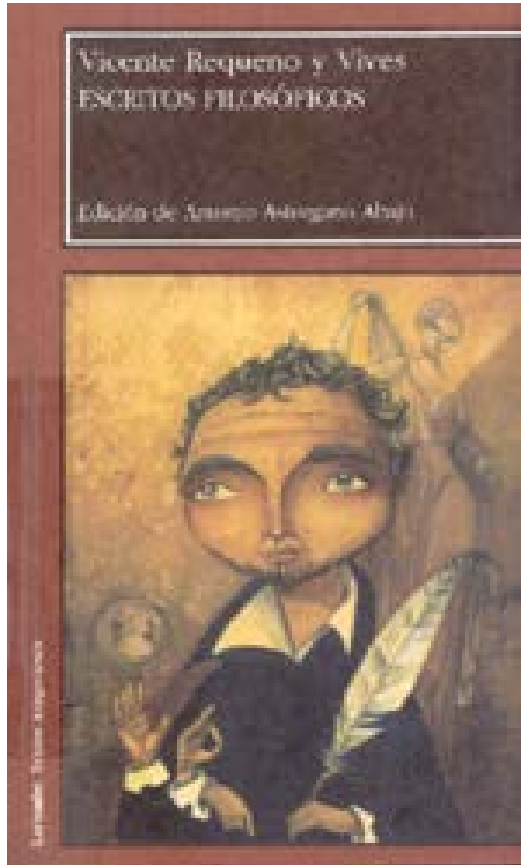

Fig. 9. Retrato imaginario de Vicente Requeno de David Guirao (2008). Aunque Requeno era pintor aficionado, no se conserva ninguna obra suya. Casanatense, que le ofreció el invasor napoleónico.

Parece increíble que un Vicente Requeno, miembro de media docena de Academias, que en el periodo de 1798-1801 se entregó en cuerpo y alma a las tareas de la Económica Aragonesa, en 1804 publicase un devocionario al culto del Sagrado Corazón de Jesús y lo dejase todo para ir a Nápoles a enseñar primeras letras en un Colegio de la restaurada Compañía.

Esta contradicción era inevitable en los ignacianos más inquietos intelectualmente, pues con un ojo miraban a sus rigurosas investigaciones históricas y artísticas, que debían estar a la altura y rigor crítico de los avances literarios y científicos del momento, y con el otro a mantener viva su auténtica vocación jesuítica. Es la misma contradicción que hallamos en personajes de la talla de otros restauradores de la Compañía, como Antonio Conca, el traductor al italiano del Viaje de España de Antonio Ponz, o Juan Andrés, padre de la Literatura Comparada, quienes colaboraron con instituciones claramente ilustradas y afrancesadas, por cierto, con la aprobación del Provincial san José Pignatelli.

Concluyamos resaltando nuevamente las virtudes de la iniciativa académica del doctor Oscar Flores, así como el acierto del Instituto de Investigaciones Estéticas de la UNAM y de la Real Academia de San Fernando en respaldar este libro sobre el P. Márquez y su época, que es un claro ejemplo de colaboración interinstitucional de cómo se debe estudiar a un relevante jesuita, que se sentía tan español como mexicano, en una época tan complicada como el tránsito del Antiguo Régimen al Liberal, y del Imperio español al independentismo iberoamericano. Muchas gracias. 